TITLE:

\title{
Alpha-fetoprotein-producing pancreatic cancer cells possess cancer stem cell characteristics.
}

\section{AUTHOR(S):}

Sasaki, Naoya; Ishii, Takamichi; Kamimura, Ryo; Kajiwara, Masatoshi; Machimoto, Takafumi; Nakatsuji, Norio; Suemori, Hirofumi; Ikai, Iwao; Yasuchika, Kentaro; Uemoto, Shinji

\section{CITATION:}

Sasaki, Naoya ... [et al]. Alpha-feto protein-producing pancreatic cancer cells possess cancer stem cell characteristics.. Cancer letters 2011, 308(2): 152-161

\section{ISSUE DATE:}

2011-09-28

\section{URL:}

http://hdl.handle.net/2433/145219

\section{RIGHT:}

(c) 2011 Elsevier Ireland Ltd.; This is not the published version. Please cite only the published version.; この論文は出版社版でありません。引 用の際には出版社版をご確認ご利用ください。 
Title

Alpha-fetoprotein-producing pancreatic cancer cells possess cancer stem cell characteristics.

\section{Abstract}

We aimed to demonstrate the existence of cancer stem cells in human pancreatic cancer, and to clarify that they are alpha-fetoprotein (AFP) producing cells. Six cell lines derived from human pancreatic cancers were examined, and AsPC-1 and PANC-1 were noted to express AFP. Single cell culture assays and xenotransplantation revealed that the AFP-producing cells had the capacity for self-renewal and differentiation, and that these cells were tumorigenic. Furthermore, they were resistant to anti-cancer agents. The ABCA12 transporter was expressed in the AFP-producing cells at a level more than twice as high as that in the non-AFP-producing cells.

The AFP-producing cells were shown to be putative pancreatic cancer stem cells. Furthermore, the expression of ABCA12 appears to be associated with drug resistance.

\section{Key words:}

\section{Cancer stem cell}

Pancreatic cancer

Alpha-fetoprotein (AFP)

ATP-binding cassette, sub-family A (ABC1), member 12 (ABCA12) 


\section{Introduction}

Recent advances in stem cell biology have revealed the existence of cancer stem cells

(CSCs) in various tumors, including leukemia [1], breast cancer [2], brain tumor [3] and pancreatic

cancer $[4,5]$. CSCs are defined as cells that possess the capacity to self-renew, to cause the heterogeneous population of cancer cells, and to initiate tumors [6]. In addition, CSCs are supposed to have long lifetime and show drug- and/or radiation-resistance, like normal tissue stem cells. These properties of the CSCs might contribute to the tumor malignancy such as rapid growth, resistance to therapy, and recurrence. Therefore, CSCs could be a new target for cancer therapies.

To investigate the characteristics of the CSCs, it is necessary to be able to successfully isolate them from the heterogeneous tumor cells. There have been several reports about the combinations of cell surface markers that can be used to isolate the CSCs from the pancreatic cancer tissues $[4,5]$. However, the functions and biological relevance of the individual markers in the tumor tissues are still unknown.

Previously, we reported the existence of CSCs in cholangiocellular carcinoma, and demonstrated that these cells were as the alpha-fetoprotein (AFP) producing cells [7]. AFP is one of the major serum proteins produced by fetal hepatoblasts. In this study, we also focused on AFP as a human pancreatic cancer stem cell marker. AFP is secreted not only by the fetal liver, but also by foregut endoderm and fetal pancreas [8]. Because CSCs show several similarities to normal tissue stem cells, we hypothesized that AFP might be expressed in human pancreatic CSCs. 


\section{Materials and methods}

\subsection{Patients}

Twenty-five patients with pathologically-confirmed pancreatic cancer who had undergone a pancreatic resection at Kyoto University Hospital in 2008, were included in this study. The patients included 10 males and 15 females, with a mean age at surgery of $66.7 \pm 7.5$ years (range, 49-77 years). Written informed consent for the use of their resected tissues was obtained from all patients in accordance with the Declaration of Helsinki. Their clinicopathological background is summarized in Supplemental Table 1.

\subsection{Generation of transgenic cell lines derived from human pancreatic cancer cells}

MIAPaCa-2, AsPC-1 and PANC-1 cells were obtained from the American Type Culture Collection (ATCC, Manassas, VA), SUIT-2 was from the Health Science Research Resources Bank

(Osaka, Japan), and KP-1NL and QGP-1 were from the Japanese Collection of Research Bioresources cell bank (Osaka, Japan). These cells were plated on cell culture dishes (BD Biosciences, Franklin Lakes, NJ), and all six cell lines were maintained in RPMI 1640 medium (Invitrogen, Carlsbad, CA) supplemented with $10 \%$ heat-inactivated fetal calf serum (FCS; ICN Biomedicals Inc., Aurora, OH), 100 U/ml penicillin (Meiji Seika, Tokyo, Japan), and $100 \mu \mathrm{g} / \mathrm{ml}$ streptomycin (Meiji Seika) at $37^{\circ} \mathrm{C}$ in a humidified atmosphere with $5 \%$ carbon dioxide.

The transgene plasmid vectors that expressed enhanced green fluorescent protein (EGFP) under the control of human AFP enhancer/promoter were generated as described previously [9]. 
The transgenic vectors were transfected using Lipofectamine 2000 (Invitrogen) according to the manufacturer's protocol. Stably transfected cells were selected in the presence of $800 \mu \mathrm{g} / \mathrm{ml} \mathrm{G} 418$ (Sigma, St Louis, MO) over a period of 30 days. The proper transgene insertion was confirmed by polymerase chain reaction (PCR). The stable transfectants were cultured in the presence of 800 $\mu \mathrm{g} / \mathrm{ml} \mathrm{G} 418$.

\subsection{Reverse transcription-PCR (RT-PCR)}

RT-PCR was performed as described previously [9]. Primers were generated for the following human genes; EGFP, AFP, and glyceraldehyde-3-phosphate dehydrogenase (GAPDH). Their sequences and the PCR conditions are summarized in Supplemental Table 2.

\subsection{Immunocytochemistry of cultured cells}

The cultured cells were fixed and stained as previously described $[9,10,11]$. An anti-human AFP rabbit antibody (DakoCytomation, Glostrup, Denmark) diluted at 1:200 was used as the primary antibody, and Alexa546-conjugated donkey anti-rabbit IgG (Molecular Probes, Eugene, OR) diluted at 1:500 was used as the secondary antibody. The stained cells were covered with Vectashield mounting medium with 4',6-diamidino-2-phenylindole (DAPI; Vector Laboratories, Burlingame, CA).

\subsection{Histology and immunohistology}


The harvested tumors were fixed in $3.3 \%$ formaldehyde (Wako, Osaka, Japan) for at least one week. After a thorough rinsing, the tissues were then embedded in paraffin and sectioned into $3 \mu \mathrm{m}$ thick sections. Hematoxylin-eosin (HE) staining was performed according to a standard protocol. In order to perform immunohistological staining for AFP, the deparaffinized and rehydrated sections were incubated in 3\% hydrogen peroxide in methanol for 10 minutes and then were incubated with the diluted anti-human AFP antibodies at $4{ }^{\circ} \mathrm{C}$ for 16 hours. Followed by thorough rinsing, the samples were incubated for 30 minutes with horseradish peroxidase-conjugated anti-rabbit IgG (Envision+ Kit/horseradish peroxidase; DakoCytomation) and then for 1 minute with 3,3'-Diaminobenzidine (DAB) substrates (DakoCytomation). Counterstaining was performed using hematoxylin.

\subsection{Flow cytometry}

The cultured cells were prepared as described previously $[9,10,11]$. Dead cells were eliminated using 7-amino-actinomysin D (7-AAD; BECKMAN COULTER, Brea, CA). The cells were analyzed and isolated using a FACSVantage SE cell sorter (BD Biosciences).

For the single-cell culture analyses, individual isolated cells were inoculated into each well of 96-well culture plates using the FACSVantage SE and the CloneCyt Plus software program (BD Biosciences). That the wells contained only one cell was confirmed using a light microscope 10 to 16 hours after cell sorting. Following cell expansion after isolation of each clone, they were subjected to flow cytometry as described previously. 


\subsection{Cell proliferation assay and anchorage-independent growth assay}

The isolated EGFP-positive and EGFP-negative cells were inoculated at a density of 1 $\times 10^{3}$ cells per well in 96-well culture plates (BD Biosciences), and then were allowed to grow for eight days. The cell numbers were determined using the 3-(4,5-dimethylthiazol-2-yl)-5-(3-carboxymethoxyphenyl)-2-(4-sulfophenyl)-2H-tetrazorium, inner salt (MTS) assay (Cell Titer 96 Aqueous One Solution Reagent; Promega, Madison, WI), according to the manufacturer's protocol. After a $1 \mathrm{hr}$ of incubation, the absorbance value was measured using a plate reader at $490 \mathrm{~nm}$.

To examine the anchorage-independent growth of the cells, $1 \times 10^{4}$ EGFP-positive and EGFP-negative cells were suspended in $2.0 \mathrm{ml}$ of $0.3 \%$ agar (Wako) supplemented with the culture medium. The cell suspension was layered over the bottom layer of $2.0 \mathrm{ml}$ of $0.6 \%$ agar. The AsPC-1 colonies that contained more than 10 cells were counted 21 days after cell sorting, and the colonies of PANC-1 cells were counted 7 days after sorting.

\subsection{Xenotransplantation}

Male 8 to 12-week-old non-obese diabetic/severe combined immune deficiency (NOD/SCID) mice (CLEA Japan, Inc., Tokyo, Japan) were utilized in the transplantation experiments. The sorted cells were washed twice with PBS after flow cytometry, and resuspended in $200 \mu \mathrm{l}$ of a serum-free medium and Matrigel (BD Biosciences) mixture (1:1 volume). Under 
general anesthesia, the cells were engrafted into the right and left dorsal areas of NOD/SCID mice subcutaneously using a 26-gauge needle. During the following 12 weeks, mice were examined periodically by visual inspection and palpation, and then they were sacrificed to extract tumors.

To investigate the composition of the tumors grown in NOD/SCID mice, serial transplantation was performed. The tumors that were generated from the single-cell-derived EGFP-positive or EGFP-negative cells were harvested. The tumor tissues were cut into small pieces by using sterile blades, and were digested with collagenase solution [12] for 60 minutes at $37^{\circ} \mathrm{C}$. After rinsing twice with PBS, the cells were analyzed using a FACSCalibur (BD Biosciences), or sorted using a FACSVantage SE. For secondary xenotransplantation, the harvested cells were cultured in G418-containing medium for at least 7 days to eliminate cells originated from the host mice. Thereafter, these cells were sorted according to the EGFP fluorescence, and then $5 \times 10^{4}$ cells of the EGFP-positive or EGFP-negative fractions were transplanted into NOD/SCID mice.

All experimental procedures utilizing animals were performed in accordance with the Animal Protection Guidelines of Kyoto University.

\subsection{Drug resistance assay}

To investigate the resistance of the cells to anticancer agents; 5-fluorouracil (5-FU; WAKO) and gemcitabine (GEM; WAKO) were added to the cultures of the EGFP-positive and EGFP-negative cells for 4 days in the presence of the anticancer agents at various concentrations 
(from $1 \times 10^{-12} \mathrm{M}$ to $\left.1 \times 10^{-4} \mathrm{M}\right)$. To determine the half-maximal effective concentration $\left(\mathrm{EC}_{50}\right)$ of these agents, MTS assays were performed after the 4 day-culture in the presence of these anticancer agents at various concentrations. The proportions of the EGFP-positive cells were analyzed with a FACSCalibur.

\subsection{Quantitative PCR (qPCR)}

The total RNA was extracted from the freshly sorted EGFP-positive and EGFP-negative cells of the AsPC-1 or PANC-1 cell lines, using an RNeasy Mini Kit (Qiagen, Chatsworth, CA) and treated with RNase-free DNase (Qiagen). The total RNA $(0.5 \mu \mathrm{g})$ was reverse-transcribed into cDNA using the RT2 First Strand Kit (SABiosciences, Frederick, MD) according to the manufacture's protocol. The qPCR assays were performed on an ABI 7500 (Applied Biosystems, Foster City, CA) using the Human Drug Transporters RT2 Profiler PCR Array and RT ${ }^{2}$ SYBR Green Master Mix (SABiosciences) according to the manufacture's protocol. The differences in mRNA expression between the EGFP-positive and EGFP-negative cells was calculated based on the DDCt method. Results were obtained from three independent trials.

\subsection{Statistical analysis}

All experiments were performed at least three times. The results are given as the means $\pm \mathrm{SD}$. The statistical analyses were performed using Student's t-test, Dunnett's multiple comparisons and 
repeated-measures analyses of variances (ANOVA). $P<0.05$ was considered to be statistically significant. All statistical tests were two-sided. 


\section{Results}

\subsection{Generation of transgenic pancreatic cancer cell lines}

RT-PCR revealed that AFP expression was observed in both the AsPC-1 and PANC-1 cell lines (Fig. 1A). The AFP-EGFP reporter vector was transfected into the six human pancreatic cancer cell lines. Only the AsPC-1 and PANC-1 cells exhibited EGFP fluorescence with fluorescent microscopy (Fig. 1B, C); the other four cell lines did not, despite the fact that they possessed the transgene in their genomic DNA (data not shown). The AsPC-1 cell line is derived from the ascites of a pancreatic adenocarcinoma patient [13], and the PANC-1 line is from a pancreatic carcinoma of ductal origin [14]. A flow cytometric analysis showed that $25.1 \pm 1.1 \%$ $(n=3)$ of the AsPC-1 cells and $6.5 \pm 1.1 \%(n=3)$ of the PANC-1 cells expressed the EGFP fluorescence (Fig. 1D, E). The sorted EGFP-positive cells expressed both EGFP and AFP mRNA, whereas the sorted EGFP-negative cells did not express either molecule (Fig. 1F), thus indicating that the EGFP-positive cells to correspond to the AFP-producing cells, and that cell sorting could be used to divide these cells according to their EGFP expression. Therefore, a further investigation of the AFP-producing cells in the both cell lines was conducted to determine whether or not they were pancreatic CSCs.

\subsection{Single cell sorting and culture assay}

Since these cell lines were heterogeneous populations, single-cell culture assays were performed in order to examine their self-renewal and differentiation capabilities. A single cell was 
sorted and cultured in a well of a 96-well culture plate. Following cell expansion by four passages, flow cytometric analyses were performed. A single EGFP-positive cell generated both EGFP-positive and EGFP-negative cell fractions (Figs. 2A, B, E, F). One EGFP-negative cell, however, produced only the EGFP-negative fraction (Figs. 2C, D, G, H). These flow cytometric findings were reproducible in three trials (four EGFP-positive clones and five EGFP-negative clones were obtained from AsPC-1, and twelve EGFP-positive and twelve EGFP-negative clones from PANC-1), and the transgene was identified in the genomic DNA of all these clones (Supplemental Figure 1). These results indicated that the EGFP-positive cells had the ability to self-renew and the ability to generate the EGFP-negative cell fraction. These results also suggested that the AFP-producing cells of both cell lines had progenitor-like characteristics.

\subsection{In vitro cell proliferation assay}

To compare the in vitro tumorigenicity between the EGFP-positive and EGFP-negative cells, cell proliferation and the anchorage-independent cell growth assays were performed. In both cell lines, the EGFP-positive cells exhibited significantly higher proliferation than did the EGFP-negative cells $(P<0.05, \mathrm{n}=3$; Fig. 3A, B $)$. Moreover, the EGFP-positive cells showed stronger colony-forming abilities (approximately 2.5-fold in AsPC-1 and 4-fold in PANC-1) in soft agar than the EGFP-negative cells did $(* P<0.005, * * P<0.001, \mathrm{n}=3$; Fig. $3 \mathrm{C}, \mathrm{D})$. These findings suggest that the EGFP-positive cells have stronger tumorigenic and malignant potentials in vitro than do the EGFP-negative cells. 


\subsection{Xenotransplantation into immunodeficient mice}

To examine the tumorigenicity in vivo, the EGFP-positive or EGFP-negative cells were transplanted subcutaneously into the backs of NOD/SCID mice. A total of 10,000 to 500 cells and the mice were observed for 12 weeks. Both the EGFP-positive and EGFP-negative cells formed tumors in NOD/SCID mice. There were no differences in the proportion of tumor formation, or the time until tumor detection between the EGFP-positive and EGFP-negative cells in the both cell lines (Supplemental Table 3).

To further examine the characteristics of the cellular differentiation potential in vivo, serial transplantation was performed. The EGFP-positive clones derived from the single-cell cultures were used as donor cells (Fig. 4A, G). The EGFP-positive and EGFP-negative fractions were isolated before the primary transplantation (Fig. 4B, H). In both cell lines, the tumors derived from the xenotransplantation of the EGFP-positive cells had both EGFP-positive and EGFP-negative cell fractions (Fig. 4D, J). In contrast, the tumors from the EGFP-negative cells generated only the EGFP-negative fraction (Fig. 4C, I). These results demonstrated the self-renewal and differentiation abilities of the EGFP-positive cells in vivo. Furthermore, the EGFP-positive and EGFP-negative cells isolated from the EGFP-positive cell-derived tumors were transplanted again at $5 \times 10^{4}$ cells into NOD/SCID mice. Both cell lines formed tumors. The tumors from the EGFP-positive cells had both the EGFP-positive and EGFP-negative cell fractions (Fig. 4F, L), and the tumors from the EGFP-negative cells had only EGFP-negative fraction (Fig. 4E, K). These 
results indicated that the EGFP-positive cells kept their self-renewal potential and ability to differentiate through several passages in vivo.

\subsection{Drug resistance assay}

The resistance of the two cell lines to 5-FU and GEM was examined. $\mathrm{EC}_{50}$ values were calculated based on MTS assays, showing that the $\mathrm{EC}_{50}$ values for 5 -FU were $5.15 \times 10^{-6} \mathrm{M}$ for AsPC-1 and $1.03 \times 10^{-6} \mathrm{M}$ for PANC-1, respectively. Those for GEM were $5.48 \times 10^{-10} \mathrm{M}$ for AsPC-1 and $4.54 \times 10^{-8} \mathrm{M}$ for PANC-1. In both cell lines, the proportions of the EGFP-positive cells increased when the whole cell populations were cultured in the presence of these anticancer agents around the $\mathrm{EC}_{50}$ (Fig. 5A-D). The number of total living cells was decreased at concentrations over the $\mathrm{EC}_{50}$. It was demonstrated that the EGFP-positive cells in both cell lines had greater drug resistance against both 5-FU and GEM.

In order to examine the mechanism responsible for the drug resistance, the expression levels of mRNA related to drug transporters that move cytotoxic substances outside the cell membrane were compared between the AFP-producing cells and the non-AFP-producing cells by qPCR assays. The ABCB1 transporter in AsPC-1 cells, and the ABCB4 transporter in PANC-1 cells, which are known multidrug resistance transporters, were expressed in the EGFP-positive cells at more than twice the levels as that in the EGFP-negative cells. In both cell lines, the ABCA12 transporter was highly expressed in the EGFP-positive cells (Fig. 5E-H). 


\subsection{Histology and immunohistology of surgical specimens}

In order to examine the AFP expression in human clinical pancreatic cancer, immunohistological assays were performed utilizing 25 surgical samples. A small number of tumor cells (less than 5\%) expressed AFP in 16 of the 25 patients with pancreatic cancer (Figure 6, A-C). However there were no AFP expressing cells in any of the examined sections of the other 9 patients (Figure 6D). The preoperative serum AFP values were below the normal range in patients who were examined. The patients' clinical background and tumor pathological features did not correlate with the AFP expression (Supplemental Table 1). 


\section{Discussion}

CSCs are thought to have many similarities to normal tissue stem cells, and are defined by their ability to self-renew, to generate heterogeneous lineages of cancer cells, and to initiate tumor growth [6]. Although many previous reports concerning the identification of CSCs used combinations of cell surface markers, we focused on AFP as a marker for pancreatic CSCs. AFP is produced by the foregut endoderm and its derivatives; the fetal liver and pancreas during the embryonic period [8]. Therefore, we hypothesized that pancreatic CSCs might express AFP if the CSCs existed in the pancreatic cancer tissues.

In this study, we transfected a plasmid vector that expressed EGFP under the control of the AFP enhancer/promoter into the six human pancreatic cancer cell lines, in order to isolate and characterize the AFP-producing cells. AFP-producing cells were found in two of the six cell lines (AsPC-1 and PANC-1). The AFP-producing cells in both cell lines were demonstrated to have the capacity for self-renewal and differentiation to generate an non-AFP-producing cell fraction in vitro and in vivo, whereas the non-AFP-producing cells did not possess these characteristics. The AFP-producing cells were also shown to have a higher proliferation activity in vitro and tumorigenicity in vivo. These results indicated that the AFP-producing cells corresponded to cancer progenitor cells in pancreatic cancer. The non-AFP-producing cells had the same tumorigenicity as the AFP-producing cells in vivo. This was probably because the tumor cell lines had, in general, a higher malignant potential than their original tumor tissues [15]. However, the non-AFP-producing cells did not generate heterogeneity concerning EGFP expression in the 
xenografted tumor tissues.

The existence of the AFP-producing cells in human clinical specimens was also demonstrated by the immunohistological analyses. A small number of tumor cells expressed AFP in 16 of the 25 patients with pancreatic cancer. There have also been several reports of pancreatic cancers with high serum AFP levels, which tend to be associated with a worse prognosis because of high rates of liver metastasis [16]. These finding suggested that AFP could be a CSC marker in clinical cases. Although there were no patients with high preoperative serum AFP values in our 25 cases, the AFP-producing pancreatic cancers might represent cases of abnormal proliferation or increased numbers of AFP-producing CSCs, which would result in a poorer prognosis.

The $\mathrm{CD} 24^{+} \mathrm{CD} 44^{+}$epithelial specific antigen $(\mathrm{ESA})^{+}[4], \mathrm{CD} 133^{+}$, and $\mathrm{CD} 133^{+} \mathrm{CXCR} 4^{+}$ cells in pancreatic cancer tissues [5] have previously been reported as pancreatic CSCs. We examined the relationship between the AFP expression and these reported cell surface markers. In the PANC-1 cells, the CD24-positive or CD133-positive cell fractions included a large part of the EGFP-positive cells. The AFP-producing PANC-1 cells were enriched in the CD $24^{+} \mathrm{CD} 133^{+}$cell fraction, confirming that CD24 and CD133 are useful for isolating CSCs in some pancreatic cancers. On the other hand, the AsPC-1 cells had neither CD24-positive nor CD133-positive cell fractions. In addition, the expression of CD44, ESA, and CXCR4 did not correlate with the AFP expression in either cell line (Supplemental Figure 2). These findings suggested that there might be several types of CSCs in various stages of differentiation, and that the profiles of cell surface markers could vary among these CSCs. The $\mathrm{CD}_{133}{ }^{+} \mathrm{CXCR} 4^{+}$cells were found in the tumor 
invasive front [5], suggesting that CSCs might change their phenotype depending on their environment.

AFP-producing cells were not found in all cell lines or all surgical specimens. These findings raise the question whether all pancreatic cancer tissues have their own CSCs. Furthermore, it remains unclear whether all pancreatic CSCs have a common marker, or whether, as mentioned above, different markers and phenotypes may occur depending on the microenvironment. Further are necessary to answer these questions. However, it would be biologically difficult to find a common marker for pancreatic CSCs because of the genetic instability of CSCs [17].

CSCs are also assumed to contribute to chemoresistance and radioresistance. This study demonstrated that the AFP-producing cells in both cell lines showed resistance to 5-FU and GEM compared to non-AFP-producing cells. The qPCR analyses revealed that the ABCA12 gene, which encodes one of the ABC transporter family proteins, was highly expressed in the AFP-producing cells of both cell lines. The expression of ABCA12 has been reported to be predominantly found in the skin and the stomach. This transporter is assumed to play a role in skin lipid homeostasis, and is highly expressed in healthy keratinocytes. Mutations in the ABCA12 gene were detected in patients suffering from lamellar ichthyosis type 2 and harlequin ichthyosis, a rare hereditary disease characterized by skin desquamation over the whole body [18]. However, there have been only a few reports about ABCA12 expression in cancer. In these reports, positive correlations were found between the expression of $\mathrm{ABCA} 12$ and the resistance of breast cancer to neoadjuvant chemotherapy [19], and a retinoblastoma cell line was reported to highly express the ABCA12 
gene [20]. Our results suggested that the ABCA12 transporter may be associated with the chemoresistance of pancreatic CSCs. It has also been postulated that some CSCs are able to enter into the G0 phase of the cell cycle, and this characteristic is also thought to enhance their chemoresistance. However, the cell cycle analyses of our cell lines showed no significant differences in the proportions of G0 phase cells between the AFP-producing and non-AFP-producing cells (data not shown).

In conclusion, the AFP-producing cells were shown to be cancer progenitor cells, or putative CSCs of human pancreatic cancer. In addition, the drug transporter ABCA12 was preferentially expressed in the AFP-producing cells, suggesting that the high expression of ABCA12 may be associated with the CSCs' resistance to anti-cancer agents. AFP and ABCA12 may represent a new prognostic marker or target for cancer therapies. 
Acknowledgements

The authors would like to thank Ms. Kimie Hamanaka for her valuable research assistance. 
References

[1]D. Bonnet, J.E. Dick, Human acute myeloid leukemia is organized as a hierarchy that originates from a primitive hematopoietic cell. Nat Med. 3 (1997) 730-737.

[2]M. Al-Hajj, M.S. Wicha, A. Benito-Hernandez, S.J. Morrison, M.F. Clarke, Prospective identification of tumorigenic breast cancer cells. Proc Natl Acad Sci U S A. 100 (2003) 3983-3988.

[3]S.K. Singh, C. Hawkins, I.D. Clarke, J.A. Squire, J. Bayani, T. Hide, R.M. Henkelman, M.D. Cusimano, P.B. Dirks, Identification of human brain tumour initiating cells. Nature. 432 (2004) 396-401.

[4]C. Li, D.G. Heidt, P. Dalerba, C.F. Burant, L. Zhang, V. Adsay, M. Wicha, M.F. Clarke, D.M. Simeone, Identification of pancreatic cancer stem cells. Cancer Res. 67 (2007) 1030-1037.

[5]P.C. Hermann, S.L. Huber, T. Herrler, A. Aicher, J.W. Ellwart, M. Guba, C.J. Bruns, C. Heeschen, Distinct populations of cancer stem cells determine tumor growth and metastatic activity in human pancreatic cancer. Cell Stem Cell. 1 (2007) 313-323.

[6]M.F. Clarke, J.E. Dick, P.B. Dirks, C.J. Eaves, C.H. Jamieson, D.L. Jones, J. Visvader, I.L. Weissman, G.M. Wahl, Cancer stem cells--perspectives on current status and future directions: AACR Workshop on cancer stem cells. Cancer Res 66 (2006) 9339-9344.

[7]T. Ishii, K. Yasuchika, H. Suemori, N. Nakatsuji, I. Ikai, S. Uemoto, Alpha-fetoprotein producing cells act as cancer progenitor cells in human cholangiocarcinoma. Cancer Lett 9 (2010) 9 .

[8]E.A. Jones, M. Clement-Jones, O.F. James, D.I. Wilson, Differences between human and mouse alpha-fetoprotein expression during early development. J Anat. 198 (2001) 555-559.

[9]T. Ishii, K. Fukumitsu, K. Yasuchika, K. Adachi, E. Kawase, H. Suemori, N. Nakatsuji, I. Ikai, S. Uemoto, Effects of extracellular matrixes and growth factors on the hepatic differentiation of human embryonic stem cells. Am J Physiol Gastrointest Liver Physiol. 295 (2008) G313-321.

[10]T. Ishii, K. Yasuchika, T. Machimoto, N. Kamo, J. Komori, S. Konishi, H. Suemori, N. Nakatsuji, M. Saito, K. Kohno, S. Uemoto, I. Ikai, Transplantation of embryonic stem cell-derived endodermal cells into mice with induced lethal liver damage. Stem Cells. 25 (2007) 3252-3260.

[11]T. Ishii, K. Yasuchika, H. Fujii, T. Hoppo, S. Baba, M. Naito, T. Machimoto, N. Kamo, H. Suemori, N. Nakatsuji, I. Ikai, In vitro differentiation and maturation of mouse embryonic stem cells into hepatocytes. Exp Cell Res. 309 (2005) 68-77.

[12]H. Azuma, T. Hirose, H. Fujii, S. Oe, K. Yasuchika, T. Fujikawa, Y. Yamaoka, Enrichment of hepatic progenitor cells from adult mouse liver. Hepatology. 37 (2003) 1385-1394.

[13]W.H. Chen, J.S. Horoszewicz, S.S. Leong, T. Shimano, R. Penetrante, W.H. Sanders, R. Berjian, H.O. Douglass, E.W. Martin, T.M. Chu, Human pancreatic adenocarcinoma: in vitro and in vivo morphology of a new tumor line established from ascites. In Vitro 18 
(1982) 24-34.

[14]M. Lieber, J. Mazzetta, W. Nelson-Rees, M. Kaplan, G. Todaro, Establishment of a continuous tumor-cell line (panc-1) from a human carcinoma of the exocrine pancreas. Int J Cancer 15 (1975) 741-747.

[15]B. Vecsey-Semjen, K.F. Becker, A. Sinski, E. Blennow, I. Vietor, K. Zatloukal, H. Beug, E. Wagner, L.A. Huber, Novel colon cancer cell lines leading to better understanding of the diversity of respective primary cancers. Oncogene 21 (2002) 4646-4662.

[16]T. Natsume, Y. Watanabe, T. Maruyama, H. Tanaka, K. Sunouchi, T. Tohma, M. Noju, Y. Muto, N. Tohnosu, T. Uehara, S. Shimizu, T. Ochiai, Y. Nabeya, Successful resection of a liver metastasis from AFP-producing pancreatic cancer resulting in long-term survival: a case report and review of literature. Pancreas. 31 (2005) 416-419.

[17]E. Lagasse, Cancer stem cells with genetic instability: the best vehicle with the best engine for cancer. Gene Ther. 15 (2008) 136-142.

[18]C. Albrecht, E. Viturro, The ABCA subfamily--gene and protein structures, functions and associated hereditary diseases. Pflugers Arch 453 (2007) 581-589.

[19]S. Park, C. Shimizu, T. Shimoyama, M. Takeda, M. Ando, T. Kohno, N. Katsumata, Y.K. Kang, K. Nishio, Y. Fujiwara, Gene expression profiling of ATP-binding cassette (ABC) transporters as a predictor of the pathologic response to neoadjuvant chemotherapy in breast cancer patients. Breast Cancer Res Treat. 99 (2006) 9-17.

[20]D. Hendig, T. Langmann, R. Zarbock, G. Schmitz, K. Kleesiek, C. Gotting, Characterization of the ATP-binding cassette transporter gene expression profile in Y79: a retinoblastoma cell line. Mol Cell Biochem. 328 (2009) 85-92. 


\section{Legends}

Figure 1.

The human pancreatic cancer cell lines that expressed EGFP under the control of the

human AFP enhancer/promoter. (A) An RT-PCR analysis of the six pancreatic cancer cell lines. (B,

C) The stable transfectants of AsPC-1 and PANC-1 cells expressed EGFP florescence in

fluorescent microscopy images (right panels). The left panels show the phase contrast images of

the same visual fields of the right panels. Original magnification: 200×. (D, E) A flow cytometric analysis revealed the AsPC-1 and PANC- 1 cells express EGFP in $25.1 \pm 1.1 \%$ and $6.5 \pm 1.1 \%$ of cells, respectively. The vertical axis indicates the 7-AAD fluorescence and the horizontal axis indicates the intensity of EGFP. (F) In both cell lines, the sorted EGFP-positive cells expressed mRNA for both EGFP and AFP (left lane), whereas the sorted EGFP-negative cells expressed neither EGFP nor AFP mRNA (right lane). GAPDH was used as an internal control and samples without reverse transcriptase (-RT) served as a negative control.

Figure 2.

Single cell sorting analyses of the AFP-producing EGFP-positive cells and non-AFP-producing EGFP-negative cells. (A, E) The individual EGFP-positive cells were sorted into each well of 96 well culture plates. The single cell indicated by the circle generated both the EGFP-positive and EGFP-negative cell fractions (B, F). On the other hand, the single EGFP-negative cell $(\mathrm{C}, \mathrm{G})$ produced only the EGFP-negative cell fraction $(\mathrm{D}, \mathrm{H})$. 
Figure 3.

Comparison of tumorigenicity in vitro between the AFP-producing EGFP-positive and non-AFP-producing EGFP-negative cells. (A, B) A comparison of the MTS assays between the two cell populations was made using repeated-measures ANOVA (both $P<0.05)$. (C, D) The graph indicates the colony numbers of the EGFP-positive and EGFP-negative cells growing in soft agar (Student's $t$-test; $* P<0.005, * * P<0.001)$. The results are presented as the means \pm SD.

Figure 4.

Flow cytometry of the serially transplanted AsPC-1 (A-F) and PANC-1 (G-L) tumors. The cultured AsPC-1 (A, B) and PANC-1 (G, H) cells derived from single cells were isolated according to their EGFP fluorescence, and transplanted into NOD/SCID mice. The tumors derived from the EGFP-positive (D and J) and EGFP-negative cells (C and I) were enzymatically digested and analyzed by flow cytometry. The EGFP-positive and EGFP-negative cells were isolated from the EGFP-positive cell-derived tumor (D and J), and were secondarily transplanted to the NOD/SCID mice. The EGFP-negative cell-derived secondary tumor had only EGFP-negative cells (E and K) but the EGFP-positive cell-derived tumor had both EGFP-positive and EGFP-negative components (F and L).

Figure 5.

The drug resistance analyses for 5-FU (A, B) and GEM (C, D). The vertical axis indicates 
the proportion of the EGFP-positive cells, and the horizontal axis indicates the concentration of the agent. In both cell lines, the number of EGFP-positive cells still increased when exposed to these drugs over the usual $\mathrm{EC}_{50}{ }^{*} P<0.05$ compared with the control in Dunnett's multiple comparisons. Quantitative PCR (qPCR) arrays for drug transporters were performed (E, G). Genes that showed mRNA expression more than twice or less than half in the AFP-producing EGFP-positive cells compared to the non-AFP-producing EGFP-negative cells are shown in (F, H). In both cell lines, the mRNA for ABCA12 was highly expressed in the EGFP-positive cells.

Figure 6.

The immunohistological analyses of human pancreatic cancer tissues. Figures $\mathrm{A}-\mathrm{C}$ were obtained from samples from three different patients with AFP-expressing tumor cells, and figure D was from one patient without AFP-expressing cells. The left row indicates the HE staining of tumor tissue; the center and right rows show immunostaining for AFP. The arrowheads indicate the cells that express AFP. Original magnifications: the left two rows, 100x; the right row, 400x. 

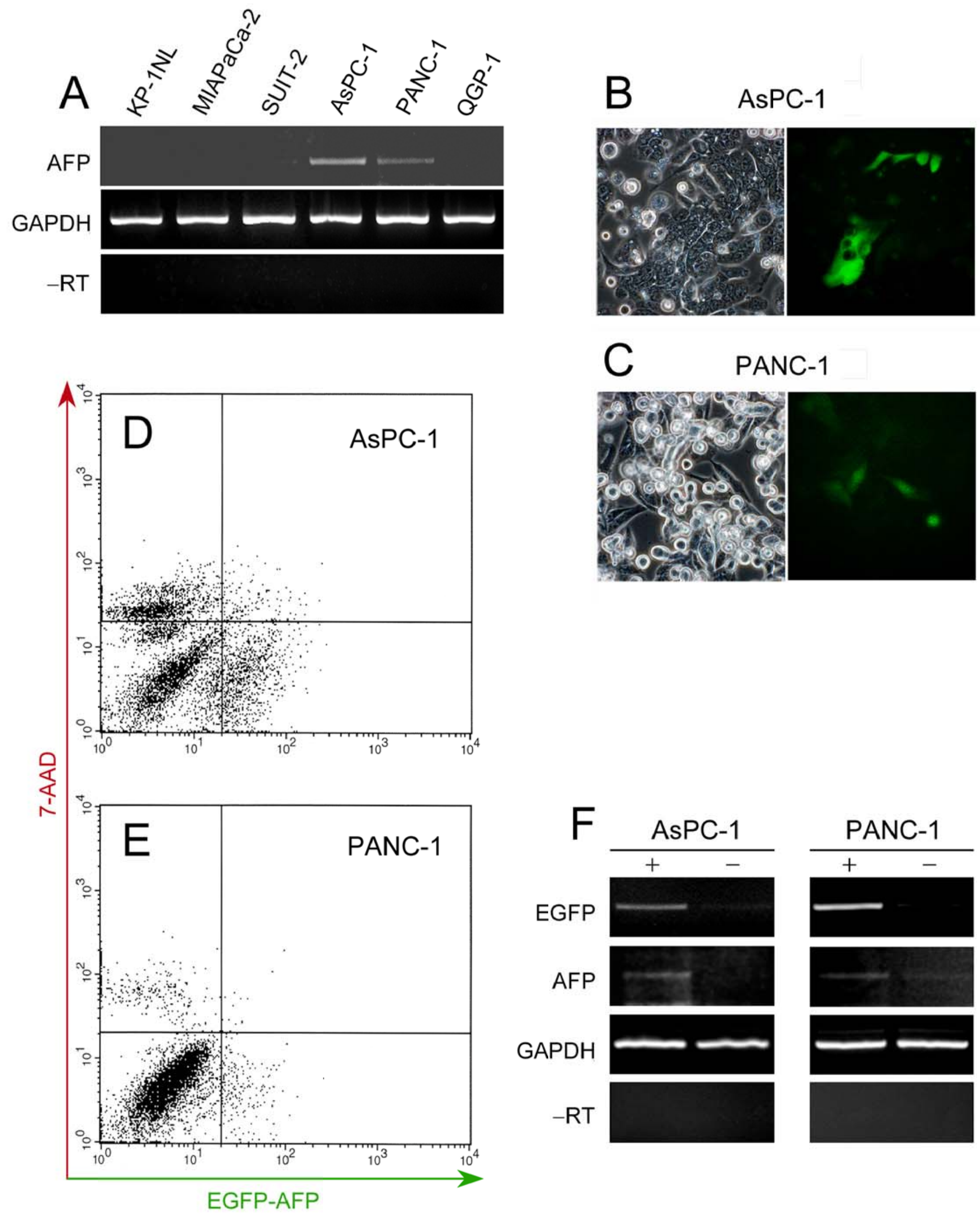


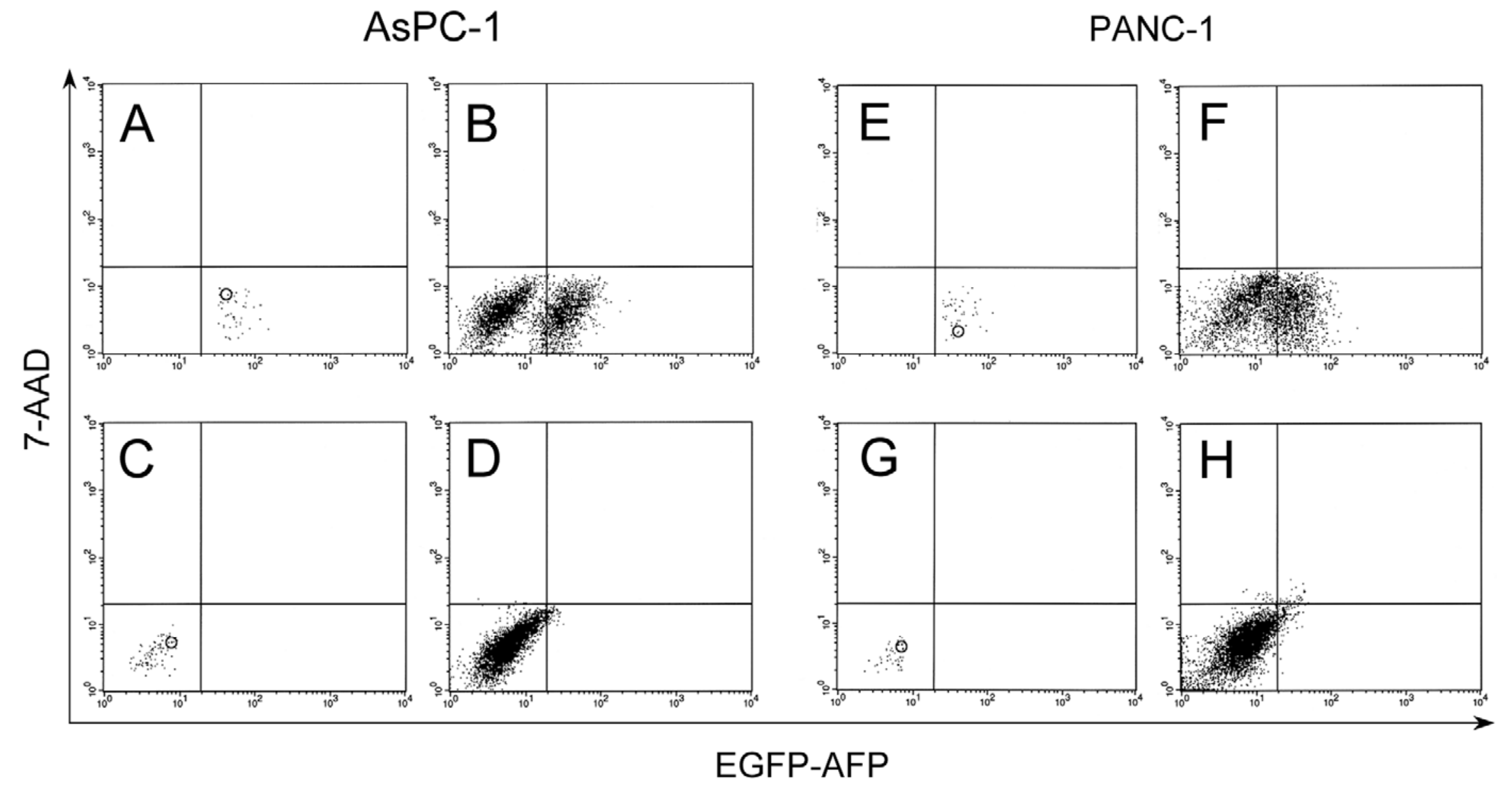



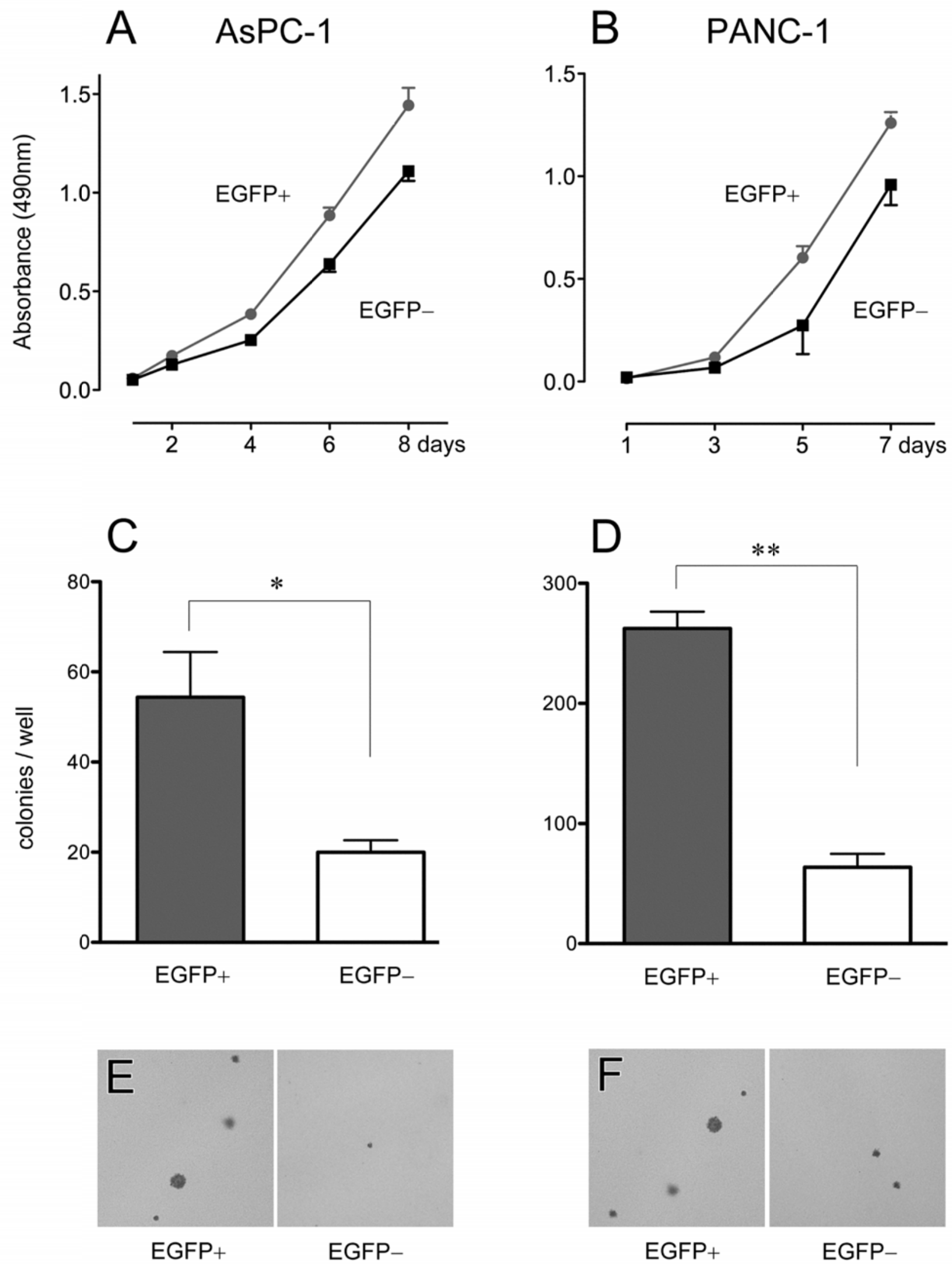
AsPC-1

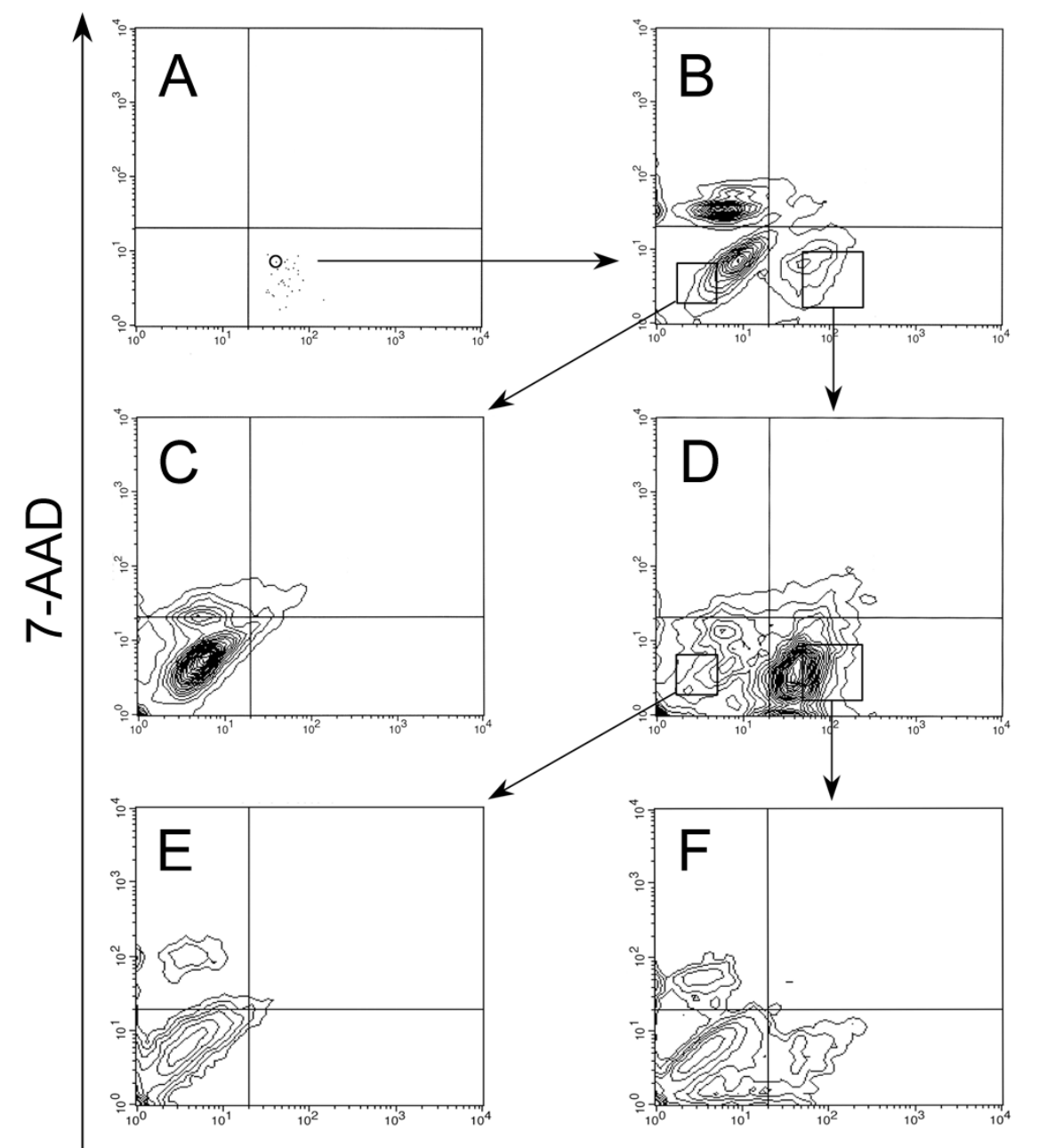

PANC-1

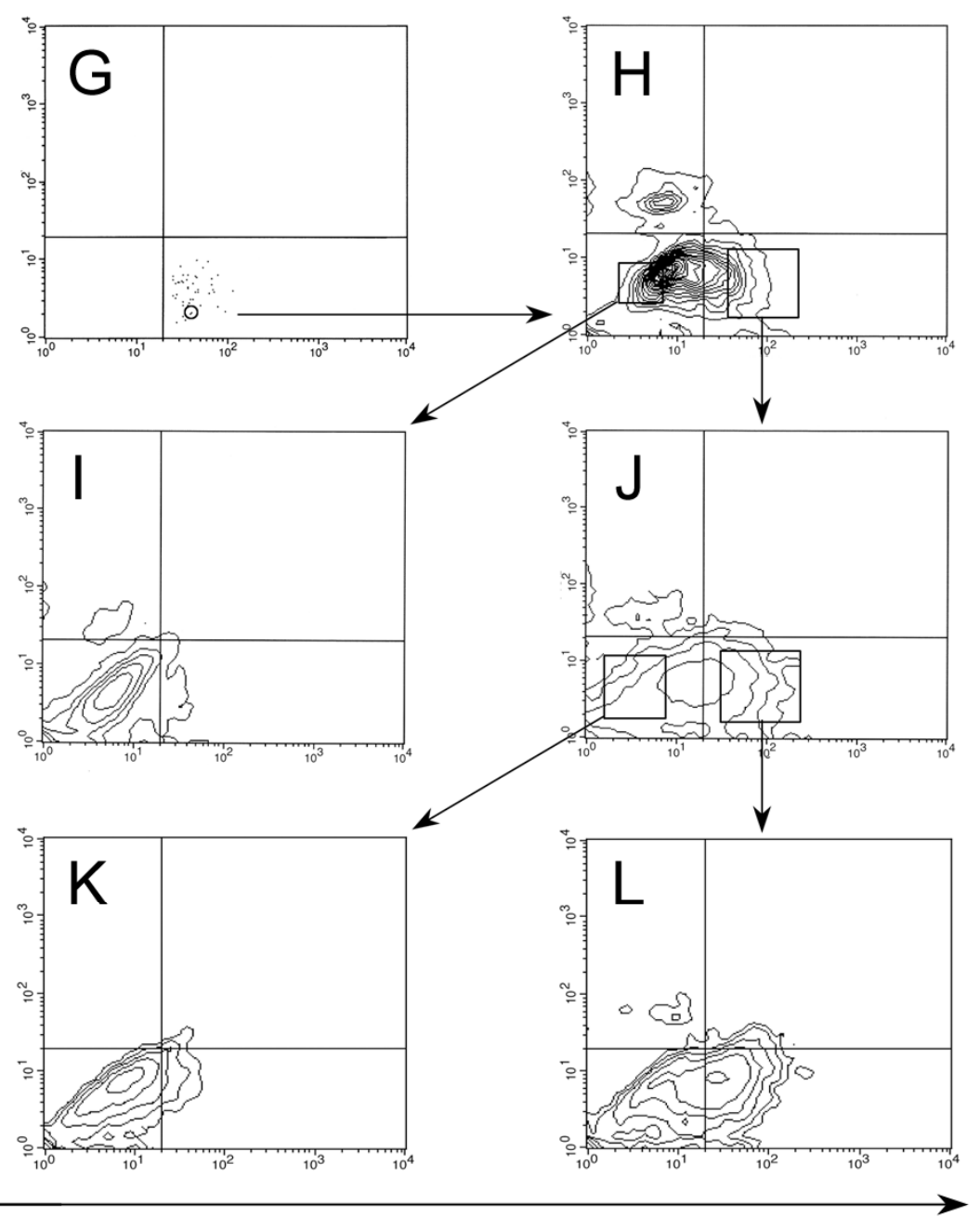

EGFP-AFP 


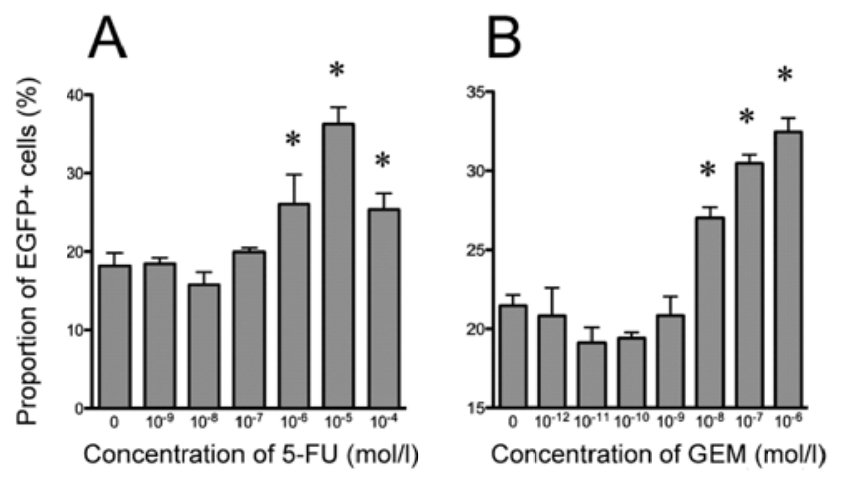

AsPC-1
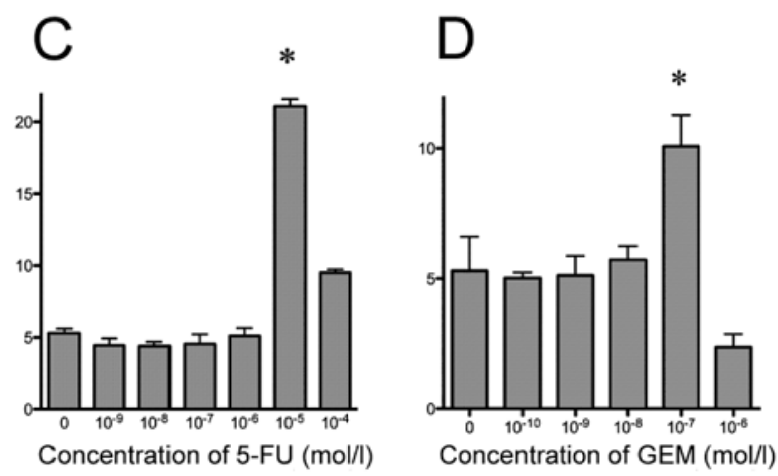

PANC-1

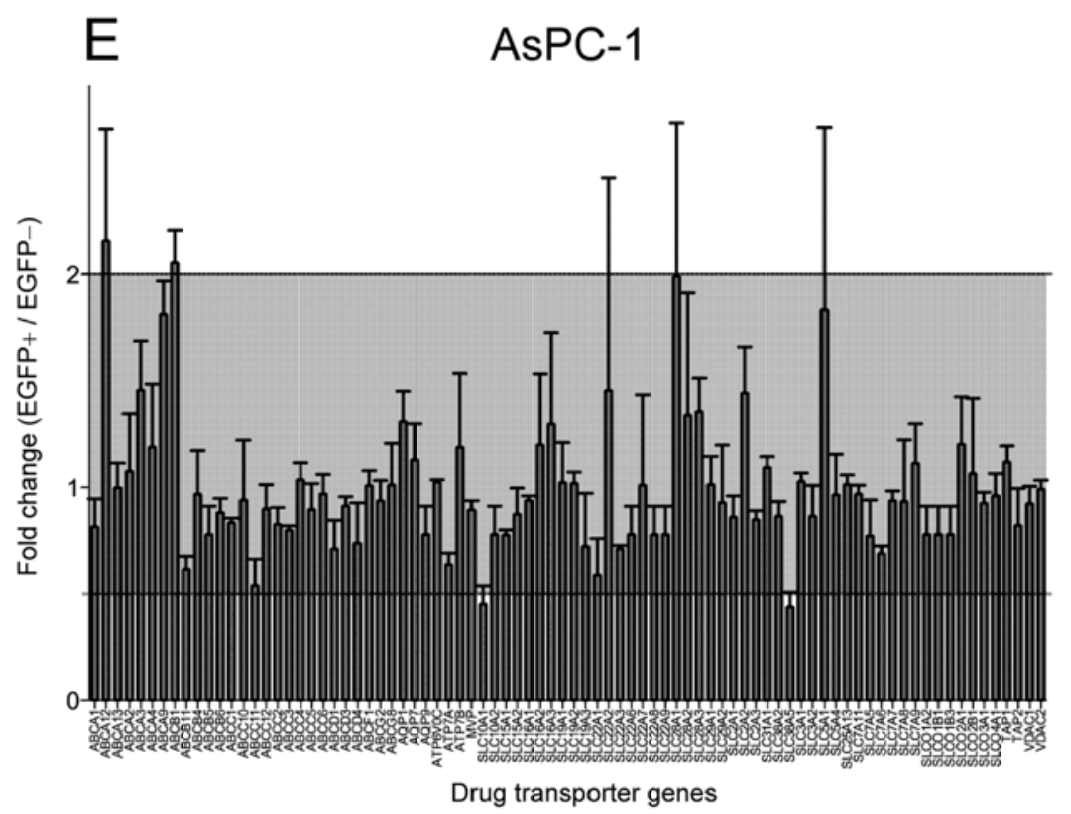

F

\begin{tabular}{cc}
$\begin{array}{c}\text { Overexpression } \\
\text { in EGFP+cells }\end{array}$ & Fold regulation \\
\hline $\mathrm{ABCA} 12$ & 2.16 \\
$\mathrm{ABCB} 1$ & 2.05
\end{tabular}

\begin{tabular}{cc}
$\begin{array}{c}\text { Overexpression } \\
\text { in EGFP- cells }\end{array}$ & Fold regulation \\
\hline SLC10A1 & 2.21 \\
SLC38A5 & 2.28
\end{tabular}

G

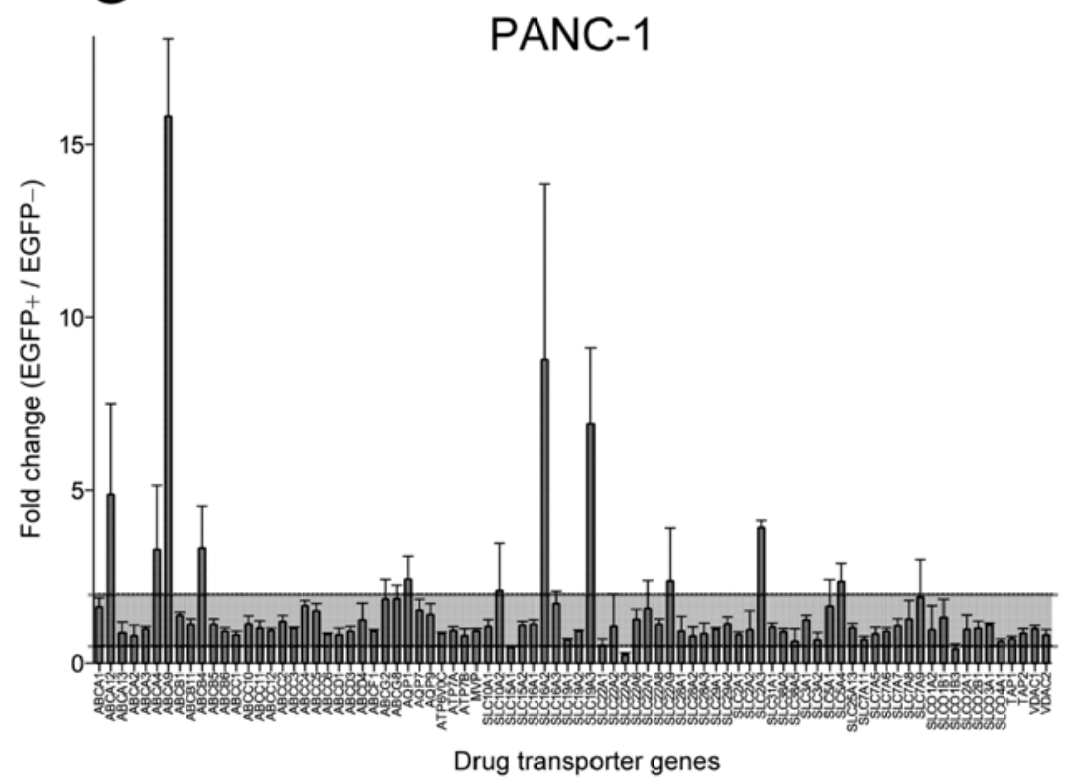

$\mathrm{H}$

\begin{tabular}{cc}
$\begin{array}{c}\text { Overexpression } \\
\text { in EGFP+ cells }\end{array}$ & Fold regulation \\
\hline ABCA12 & 4.87 \\
ABCA4 & 3.28 \\
ABCA9 & 15.82 \\
ABCB4 & 3.33 \\
AQP1 & 2.43 \\
SLC10A2 & 2.10 \\
SLC16A2 & 8.77 \\
SLC19A3 & 6.92 \\
SLC22A9 & 2.38 \\
SLC2A3 & 3.93 \\
SLC5A4 & 2.36 \\
\hline
\end{tabular}

\begin{tabular}{cc}
$\begin{array}{c}\text { Overexpression } \\
\text { in EGFP- cells }\end{array}$ & Fold regulation \\
\hline SLC15A1 & 2.20 \\
SLC22A3 & 4.10 \\
SLCO1B3 & 2.44 \\
\hline
\end{tabular}



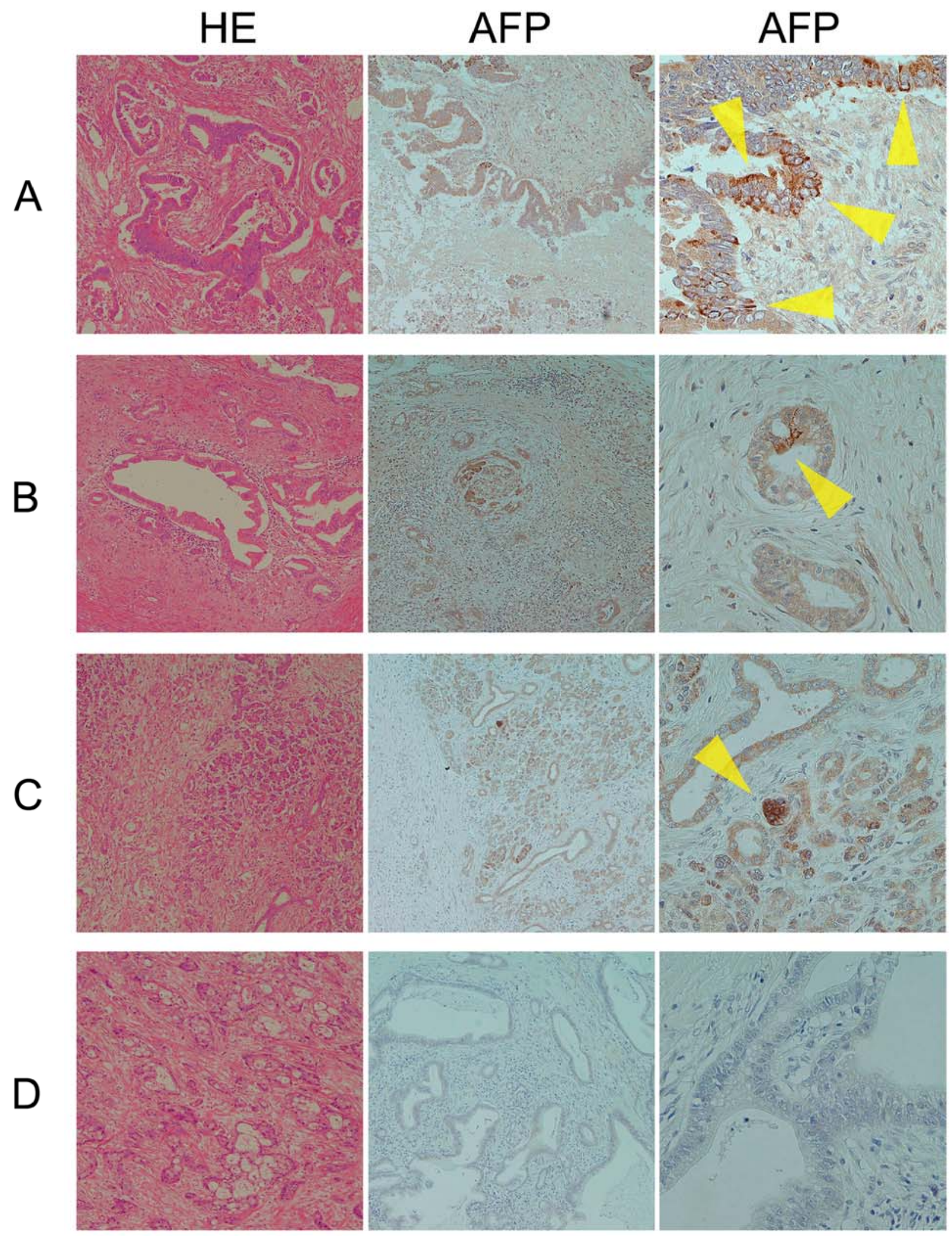


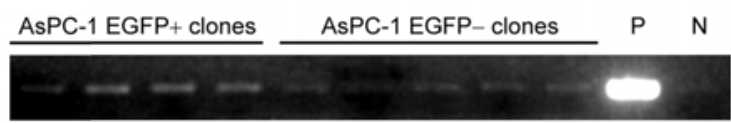

PANC-1 EGFP+ clones

P N

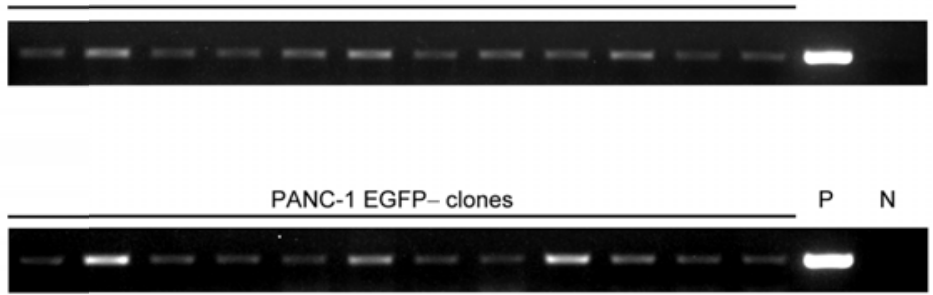

Supplemental Figure 1

Genomic PCR of clones generated from single cell cultures. The genomic DNA was extracted using DNAzol Reagent (Invitrogen) according to the manufacturer's protocol. All the AsPC-1 and PANC-1 clones generated from single cell cultures were demonstrated to have a part of the transfected genes; EGFP. The primer sequences and PCR conditions are listed in Supplemental Table 2. 
A

AsPC-1
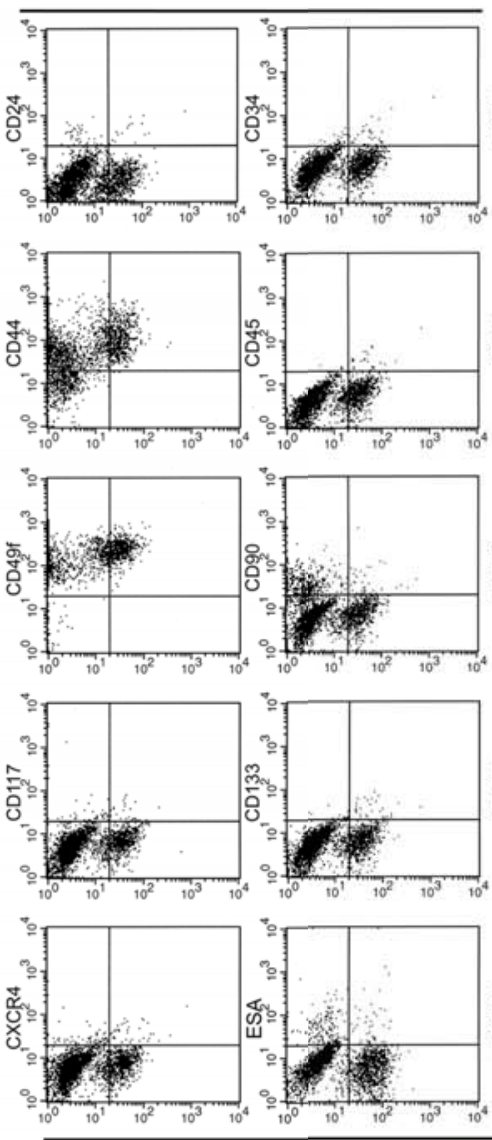

B

PANC-1
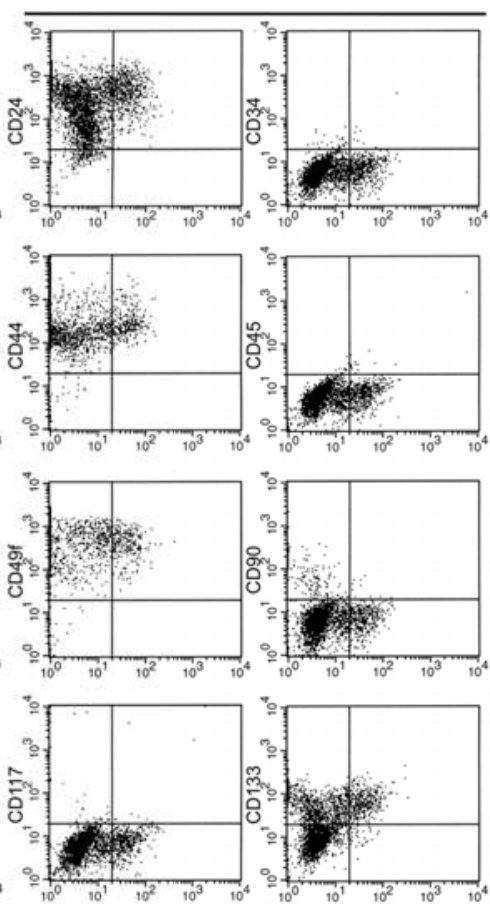

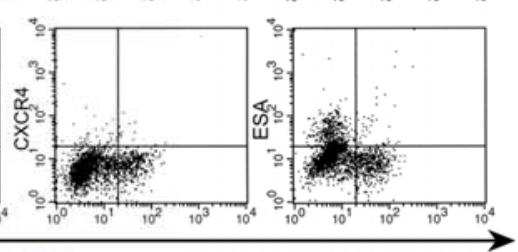

EGFP

PANC-1

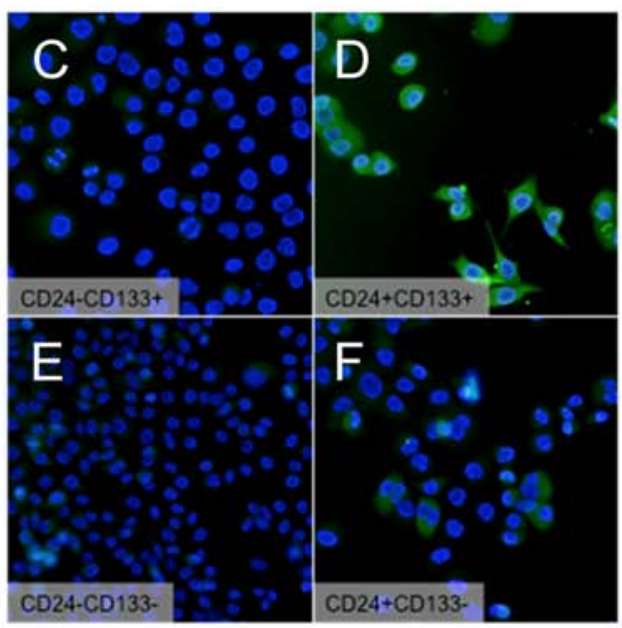

AFP/DAPI

\section{Supplemental Figure2}

Flow cytometric analyses to show the relationship between EGFP-AFP and other stem cell surface markers in AsPC-1 (A) and PANC-1 (B). These cells were analyzed and isolated using a FACSVantage SE cell sorter. The phycoerythrin-conjugated (PE) antibodies were; anti-CD24, anti-CD34, anti-CD44, anti-CD45, anti-CD49f, anti-CD90, anti-CD117, anti-CD133

(MiltenyiBiotec, BergischGladbach, Germany), anti- epithelial cell adhesion molecule (EpCAM, same as epithelial specific antigen, ESA; Abcam, Cambridge, UK), and anti- CXC chemokine receptor 4 (CXCR4; R\&D systems, Minneapolis, MN). Unless noted, antibodies were purchased from BD Biosciences. The vertical axis indicates the expression of the individual markers and the horizontal axis represents the intensity of EGFP. In PANC-1, the expression of EGFP was observed to positively correlate with CD24 and CD133.

AFP immunocytochemistry of non-transgenic PANC-1 cells sorted according to the expression of CD24 and CD133(C-F).

The CD24+ CD133+ fraction was enriched with the AFP-positive cells (D). 
Supplemental Table 1

\begin{tabular}{lc}
\hline Age at surgery & $66.7 \pm 7.5$ \\
Gender & \\
Male & $10(6)$ \\
Femal & $15(10)$ \\
Preoperative chemotherapy & \\
$\quad+$ & $3(2)$ \\
$\quad-$ & $22(14)$ \\
Operative procedure & \\
Pancreatoduodenectomy & 19 \\
Distal pancreatectomy & 4 \\
Total pancreatectomy & 2 \\
Tumor location & \\
Head & 20 \\
Body & 5 \\
Tail & 0 \\
Tumor size (mm) & $30.5 \pm 15.2$ \\
Pathology & \\
Invasive ductal carcinoma & \\
-tubular adenocarcinoma & \\
$\quad$ well differentiated & \\
$\quad$ moderately differentia & $16(11)$ \\
-poorly differentiated & $2(1)$ \\
-adenosquamous carcinc & $1(1)$ \\
IPMN * & $1(0)$ \\
TNM stage & \\
IA & \\
IB & \\
IIA & \\
IIB & \\
III & \\
IV & \\
Preoperative serum AFP (ng/ml) & \\
< 15 ng/ml & \\
I 15 ng/ml & \\
Unknown & \\
\hline
\end{tabular}

The clinicopathological background of the patients that underwent pancreatic resection at Kyoto University Hos] in 2008.

The numbers in parentheses indicate those of patients wi the AFP-producing cells in their tumor tissues. 
Supplemental Table 2

PCR primer sequences and PCR conditions

\begin{tabular}{cllcc}
\hline Gene Symbol & Forward Primer & Reverse Primer & Annealing Temperature & PCR Cycles \\
\hline AFP & 5'-GGGAGCGGCTGACATTATTA & 5'-CCCTCTTCAGCAAAGCAGAC & $60^{\circ} \mathrm{C}$ & 30 \\
EGFP & 5'-AAGCAGCACGACTTCTTCAA & 5'-CGGCCATGATATAGACGTTG & $60^{\circ} \mathrm{C}$ & 25 \\
GAPDH & 5'-CGAGATCCCTCCAAAATCAA & 5'-GTCTTCTGGGTGGCAGTGAT & $60^{\circ} \mathrm{C}$ & 25 \\
\hline
\end{tabular}

GAPDH; glyceraldehyde-3-phosphate dehydrogenase 


\begin{tabular}{ccccc} 
& & $1 \times 10^{4}$ & $5 \times 10^{3}$ & $5 \times 10^{2}$ \\
\hline \multirow{2}{*}{ AsPC-1 } & EGFP + & $5 / 6$ & $3 / 3$ & $1 / 3$ \\
& EGFP - & $5 / 6$ & $3 / 3$ & $0 / 3$ \\
\hline \multirow{2}{*}{ PANC-1 } & EGFP + & $4 / 5$ & $8 / 9$ & $3 / 6$ \\
& EGFP - & $4 / 6$ & $7 / 9$ & $3 / 6$ \\
\hline
\end{tabular}

The table shows the tumor incidence of the cell transplanted mice The top row indicates the cell numbers of transplantation per mouse. The numerators indicate the number of mice that had tumors, and the denominators indicate the number of mice that received cell transplantations. The tumorigenicity between the EGFP-positive and the EGFP-negative cell transplanted group wi analyzed using Fisher's exact test. Although $P<0.05$ was considered to be statistically significant, no significant difference was observed in the tumorigenicity between the AFP-producing 